\title{
DMC and VMC Calculations of the Electric Dipole Moment and the Ground-State Total Energy of Hydrazine Molecule Using CASINO- Code
}

\author{
Sylvester A. Ekong ${ }^{1 *}$, Vincent A. Akpan², Osarodion Ebomwonyi ${ }^{3}$ \\ ${ }^{1}$ Department of Physics and Energy Studies, Achievers University, P.M.B. 1030 Owo, Ondo State, \\ Nigeria. \\ ${ }^{2}$ Department of Physics Electronics, Federal University of Technology, P.M.B. 704 Akure, Ondo \\ State, Nigeria. \\ ${ }^{3}$ Department of Physics, University of Benin, P.M.B. 1155 Benin City, Nigeria. \\ *E-mail address: ani_sly@yahoo.co.uk
}

Keywords: Hydrazine $\left(\mathrm{N}_{2} \mathrm{H}_{4}\right)$ molecule, QMC, VMC, DMC, CASINO-Code.

\begin{abstract}
Quantum Monte Carlo (QMC) calculations of the electric dipole moment and ground-state total energy of hydrazine $\left(\mathrm{N}_{2} \mathrm{H}_{4}\right)$ molecule using CASINO-code have been carried-out by employing the VMC and DMC techniques. The optimization of the Slater-Jastrow trial wavefunction was done using variance-minimization scheme. The simulations require that the configurations must evolve on the time scale of the electronic motion, and after equilibration, the estimated effective time-step be obtained. In this study, the electric dipole moment of $\mathrm{N}_{2} \mathrm{H}_{4}$ molecule was calculated using only the DMC technique; and a value of $2.0 \mathrm{D}$ which is in good agreement with the experimental value of $1.85 \mathrm{D}$ was obtained. On the other hand, the ground-state total energy of $\mathrm{N}_{2} \mathrm{H}_{4}$ molecule was calculated using both VMC and DMC methods. It was observed that the result obtained from the $\mathrm{VMC}$ technique agrees very-well with the best theoretical value [17], but the DMC technique gave a ground-state total energy lower than all other theoretical values in literature, suggesting that the DMC result of $-111.842774 \pm 0.00394$ a.u. may be the exact ground-state total energy of hydrazine molecule. The calculated values of electric dipole moment and ground-state total energy in this work are compared with the available experimental values and the values reported by different workers. Reasonably good agreement has been obtained between them in the required order of chemical accuracy.
\end{abstract}

\section{INTRODUCTION}

The nitrogen atom is present in a large number of functional groups contained in many organic and inorganic compounds. There are, however, comparatively few compounds containing the singly bonded $>\mathrm{N}-\mathrm{N}<$ fragment, because such systems are destabilized by the repulsion of nitrogens' lone electron pairs. The parent compound, hydrazine $\left(\mathrm{N}_{2} \mathrm{H}_{4}\right)$, is kinetically stable, but thermodynamically unstable [1]. Hydrazine is one of the simplest nitrogen compounds and an important rocket fuel; and is extremely toxic, but is at the same time a very reactive and efficient reagent, which in combination with Dinitrogen Tetroxide as fuel oxidant gives a missile a much faster response time than all other propellants used before [2]. The properties of hydrazine are of interest due to their biological activities and their use as metal extracting agencies. It is also known that the carcinogenic and toxicologic consequences associated with inhalation or ingestion of Hydrazine include damage to internal organs, creation of blood abnormalities, irreversible deterioration of the nervous system, and even teratogenic and mutagenic effects [3]. Hydrazine $\left(\mathrm{N}_{2} \mathrm{H}_{4}\right)$ as strong reducing agent used both in thermal and nuclear power plants because of its ability to eliminate dissolved oxygen and protect structural materials against corrosion [4-6] has its oxygen scavenging properties that can prevent the formation of iron hydroxide and other rusts in the heat transport system [4]. Along with their use as high-energy propellants in thrusters for rockets, satellites and space shuttles, and as a monopropellant in gas turbine generators [7-9], the multipurpose chemical reagent - hydrazine - 
also have a number of commercial applications, including its role as essential building blocks in the synthesis of various polymers, pesticides, pharmaceuticals and chemotherapeutic agents; and are used as explosives to military fuel cells, in metal finishing (nickel plating), in boiler waterfeed deoxygenation, in photographic development etc.[10]. As it is very toxic and unstable, very little is currently known about its electric dipole moment. Due to the crucial and safety issues associated with the handling of Hydrazine $\left(\mathrm{N}_{2} \mathrm{H}_{4}\right)$, molecular modeling and simulation can play a particularly important role for the investigation of the energetic and non-energetic (-electric dipole moment-) properties of this molecule. According to a recent study however, classical molecular simulations, comprising molecular dynamics (MD) and Monte Carlo (MC) calculations are still uncommon for Hydrazine and its derivatives in the scientific literature [2] especially for the accurate description of electric dipole moment of hydrazine molecule.

One of the first theoretical works to study the electric dipole moment of hydrazine molecule based on the data prepared by Maryott and Buckley in 1953 was the work of Nelson Jr. et al. [11]. They calculated the hydrazine's electric dipole moment based on their principal methods of dipole moment measurement and obtained a value of $1.75 \mathrm{D}$, which though in good agreement, underestimated the experimental value of $1.85 \mathrm{D}$ reported by Seddon et al. [12]. However, due to the advent of more powerful computational techniques and methods, researchers have shown more interest in calculating the electric dipole moment of hydrazine to the nearest accuracy, all in an attempt to bridge the gap between experiment and theory. Recently, Kaczmarek et al. [13] calculated the electric dipole moment of hydrazine molecule using molecular dynamics and obtained a static equilibrium value of 2.22D which differs from the experimental value [12] by 0.37D. More recently, in 2014 Elts et al. [2] calculated from their molecular model the electric dipole moment of hydrazine molecule as $2.25 \mathrm{D}$ which agrees well with the work of [13] but differs from experiment [12] by $0.4 \mathrm{D}$, which is much more farther from experiment. However, the electric dipole moments calculated from both [13] and [2] overestimated the experimental value with a deviation of $20.0 \%$ and $21.6 \%$ respectively. These are relatively high.

Motivated by the astrophysical importance of hydrazine (N2H4), there have been many ab initio theoretical studies and spectroscopic studies on the ground-state total energy of hydrazine molecule using various techniques [14-18]. The historical account of the accurate calculation of the ground-state total energy of hydrazine (N2H4) molecule started in 1970 with work of Yamabe et al., who used a semi-empirical ASMO SCF method to study hydrazine (N2H4) molecule and the electronic transition energy of the molecule [14]. They reported the dependence of the ground-state total energy and the interaction of the lone pairs on the dihedral angle and an energy-component analysis of the molecule. They observed that the main factors contributing to the instability of the ground-state of $\mathrm{N} 2 \mathrm{H} 4$ are the electrostatic interaction term and the core-core repulsion term [14]. In 1986, Peel [15] in an ultraviolet photoelectron spectroscopy studies calculated the ground-state cations of hydrazine molecule and observed the adiabatic and vertical ionization energies with reasonable accuracy. The ground-state total energy calculated was -111.5544 a.u.; and it was observed that the deviation in the calculated ground-state total energy is a measure of the considerable change in geometry accompanying ionization. Later in 1993, Schlegel and Skancke [17] calculated the ground-state total energy of hydrazine $(\mathrm{N} 2 \mathrm{H} 4)$ at the MP4/6-31G** level. The value was modified by corrections for diffuse functions (from an MP4/6-311+G** computation), higher polarizations on non-hydrogens (MP4/6-3 1 lG(2df,p)), correlation beyond fourth-order perturbation theory (QCISD(T)/6-31 $\left.1 \mathrm{G}^{* *}\right)$, and higher level contributions. Their calculated value of -111.68045 a.u. was the best as at the time [17]. More recently, in 2005 Pires and Jorge [18] employed the IGCHF method to calculate the ground-state total energy of hydrazine molecule and obtained a value of 111.22978 a.u., thereby underestimating the ground-state total energy of hydrazine molecule calculated by [17] with a value of -0.45067 a.u. It is true that significant progress has been made in the past from other authors [16] in this direction to calculate the ground-state total energy of hydrazine but their results are in variance with recent theoretical calculations; hence further 
progress is expected. Therefore, an accurate theoretical determination of hydrazine ground-state total energy is clearly desirable.

In this paper, we report the electric dipole moment and the ground-state total energy of hydrazine (N2H4) molecule, calculated using quantum Monte Carlo (QMC) CASINO-code by employing the restricted Hartree-Fock (RHF) scheme with Gaussian basis sets. The reason for the significant deviations in the results of [13] and [2], for the electric dipole moment could be attributed to the fact that the authors used variational techniques which goes a long way to limiting the accuracy of the zero-point energy due to the necessity of guessing the trial wavefunction. On the basis of this, we have tackled the problem here by using the DMC method which requires an optimized trial wave-function as a sampling function. In fact, reported studies show that DMC, even with trial wave functions based on single Slater configuration can reproduce experimental values with very high accuracy [19]. In this study, we have also employ two QMC techniques (VMC and DMC technique) to calculate the ground-state total energy of $\mathrm{N} 2 \mathrm{H} 4$ molecule, and our results are presented in Table 1 and Table 2 respectively.

The rest of this paper will be as follows: First, the VMC and DMC methods are briefly introduced. Secondly, we explain the computational details. Thirdly, the results and discussion are presented. Finally, the paper ends with concluding remarks.

\section{THEORETICAL METHODS}

A brief overview of variational Monte Carlo (VMC) and diffusion Monte Carlo (DMC) methods are hereby presented. For more detailed discussion on these techniques, the following reviews can be consulted: Refs [20-23].

\subsection{Variational Monte Carlo (VMC) method}

The variational QMC technique is implemented in the use of the CASINO-code in this review, for the calculation of the ground-state energy of the hydrazine molecule, by employing the RHF (Restricted Hartree-Fock) method. The RHF method involves where the atoms or molecules is a closed-shell system with all orbitals (atomic or molecular) doubly occupied. It is a variant of Hartree-Fock theory for open shell molecules. It uses doubly occupied molecular orbitals as far as possible and then singly occupied orbitals for the unpaired electrons. The foundation of the RHF method were first formulated by Roothaan [24] and then extended by other authors [25, 26].

An efficient QMC variant is the variational QMC method (VQMC). Here the Rayleigh-Ritz quotient [27]:

$$
E_{T}=\frac{\left\langle\psi_{T}|H| \psi_{T}\right\rangle}{\left\langle\psi_{T} \mid \psi_{T}\right\rangle}=\frac{\int \psi_{T}^{2} \frac{H \psi_{T}}{\psi_{T}} d \tau}{\int \psi_{T}^{2} d \tau}
$$

is evaluated with Monte Carlo integration. The energy $E_{T}$ is variational: $E_{T} \geq E_{0}$. Usually, but not necessarily, $\psi_{T}^{2}$ is sampled with the Metropolis algorithm [28]. In the current VMC applications, the generalized Metropolis algorithm [29] is used, thereby allowing directed finite time steps such as diffusion step from the importance-sampled DMC algorithm. This not only increases the efficiency of the VMC method considerably, but it also makes DMC and VMC algorithms very similar, with VMC being more efficient and less accurate. Because of its efficiency, VMC is the method used for the largest QMC applications.

\subsection{Diffusion Monte Carlo (DMC) method}

DMC is a stochastic projector method for solving the imaginary-time many-body Schrödinger equation, where the state with the lowest energy is projected out of a trial wavefunction $\Psi_{\mathrm{T}}$ defined in a space spanned by the eigen-states of the Hamiltonian of the system as:

$$
\psi_{T}=\sum_{i=0} a_{i} \phi_{i},
$$


where $\left\{\phi_{i}\right\}$ is the set of eigen-states with eigenvalues $\left\{E_{i}\right\}$, i.e. $H \phi_{i}=E_{i} \phi_{i}$. Assuming that $a_{0} \neq 0$, the state with the lowest energy $\left(\phi_{0}\right)$ is singled out by applying the projection operator, $e^{-\left(H-E_{T}\right) t}$ to the trial wave function as

$$
\phi_{0}=\lim _{t \rightarrow+\infty} e^{-\left(H-E_{T}\right) t} \psi_{T} .
$$

only if $E_{T}$ becomes $E_{0}$; and the parameter t (imaginary time) in the projection operator is a positive real number. For higher efficiency, however, the importance sampling by $\psi_{T}$ is introduced so that the resulting product, $f(\mathbf{R}, t)=\phi_{0}(\mathbf{R}, t) \psi_{T}(\mathbf{R})$, obeys the integral equation:

$$
f(\mathbf{R}, t+\tau)=\int d \mathbf{R}^{\prime} \tilde{G}\left(\mathbf{R}^{\prime} \rightarrow \mathbf{R}, \tau\right) f\left(\mathbf{R}^{\prime}, t\right),
$$

where

$$
\tilde{G}\left(\mathbf{R}^{\prime} \rightarrow \mathbf{R}, \tau\right)=\left\langle\mathbf{R}\left|e^{-\left(H-E_{T}\right)}\right| \mathbf{R}^{\prime}\right\rangle \psi_{T}(\mathbf{R}) \psi_{T}\left(\mathbf{R}^{\prime}\right)^{-1}
$$

is the Green's function with the propagation time-step $\tau$, and $\mathbf{R}$ and $\mathbf{R}^{\prime}$ denotes electronic system configurations. In the long time limit, $f(\mathbf{R}, t)$ converges in the following form:

$$
\lim _{t \rightarrow \infty} f(\mathbf{R}, t)=\phi_{0}(\mathbf{R}) \psi_{T}(\mathbf{R}) \text {. }
$$

The simulation above begins with the sampling of $\left|\psi_{T}(\mathbf{R})\right|^{2}$ by an ensemble of configurations (or walkers) which subsequently evolve in accordance with the Green's function $\tilde{G}\left(\mathbf{R}^{\prime} \rightarrow \mathbf{R}, \tau\right)$ into the product of the ground-state $\phi_{0}(\mathbf{R})$ and the trial wave function $\psi_{T}(\mathbf{R})$. An analysis of the Green's function shows that the evolution of the configurations can be represented by stochastic realization of processes such as diffusion, branching and drift. Once the simulation reaches equilibration, calculations of the required expectations are carried out.

Unfortunately, application of this procedure to an electronic system leads to the well-known fermion sign problem. It stems from the fact that the function $f(\mathbf{R}, t)=\phi_{0}(\mathbf{R}) \psi_{T}(\mathbf{R})$ is not positive for the entire configuration space due to the antisymmetry of fermionic wave functions. One possible way of how to circumvent the sign problem is to force the ground-state $\left(\phi_{0}\right)$ to adopt the nodes of the trial wave function $\left(\psi_{T}\right)$. In this way, the product of the ground-state $\left(\phi_{0}\right)$ and the trial wave function $\left(\psi_{T}\right)$ will be non-negative in the entire configuration space. This approach which is known as the fixed-node approximation ameliorates the inefficiency of inherency to the fermion signs at the cost of introducing the fixed-node bias. The fixed-node bias vanishes as the nodes of the trial wave function get closer to the exact nodes. Hence, the quality of the nodes of a trial wave function is fundamental in FN-DMC. Since the state projected out in the fixed-node DMC is the state with the lowest energy of a given nodal symmetry, the required ground-state can be projected out by the fixed-node DMC if the nodal surface imposed by the trial wave function is the same as the nodal surface of the exact state [20]. The nodal constraint enforced by the fixed-node approximation enables DMC to effectively calculate the electric dipole moment of the system. The functional form of $\left(\psi_{T}\right)$ is chosen to contain a number of parameters whose values are obtained by stochastic optimization. Although the accuracy of the DMC relies on the Slater component, the Jastrow factor which captures dynamic correlation and a back flow transformation which allows further variations in the nodal surface are also vital for the efficiency of the simulation since it helps to reduce fluctuations and also the computational cost.

\section{COMPUTATIONAL DETAILS}

In this work, the simulations were done using the QMC software package, CASINO-code [23]. The CASINO-code simulation was generated for a dual purpose, and thereby used for calculating the ground-state energies as well the electric dipole moment of hydrazine molecule, with the time step (for dtdmc) set at 0.002. In our optimization, we used Gaussian basis sets by considering all electrons for all atom types and we employed the restricted Hartree Fock (RHF) technique in this case. The DMC calculation of the ground-state energy was carried-out from VMC 
optimization to confirm that the optimized equilibration was stable. The VMC steps serves as an input parameter to the $\mathrm{DMC}$, corresponding to the total number of particle configurations for which the ground-state energy is calculated. The correlated wave-function from VMC is then optimized by DMC using the variance-minimization method to obtain an efficient and more accurate convergence of the ground-state energy. In order to calculate the electric dipole moment by means of QMC, the energy calculation was carried out separately. One of the most important steps in our QMC calculations was to obtain suitable trial wave function. Following the generation of the Slater part, the optimization of the Jastrow function containing one-body and two-body explicit correlation terms was carried out. The optimization of the Jastrow function was as important as obtaining the Slater part since inadequacy in the Jastrow can increase the locality approximation bias. In our present paper, up to 28 Jastrow variational parameters were used and their optimization was performed with the variance-minimization scheme of [30] in the frame work of variational Monte Carlo (VMC), another variant of QMC.

The CASINO - code used was run on a Linux based operating system (Ubuntu environment) having a working Fortran 90 compiler.

\section{RESULTS AND DISCUSSION}

The optimized dmc steps generated give rise to new configurations of electrons and nuclei at each move and because of the difference in inter-particle separation, each of these configurations will be different. The orientational polarization of the molecule may also be a contributory factor to this amongst others. The correct expectation value of the electric dipole moment of the hydrazine molecule in this work is the average electric dipole moment of thousands of these configurations. Figures 1(a), and Figure 1(b), show the graphs of the dorsal view and lateral view results of the DMC run for hydrazine molecule, generated from 40,000 configurations. The number of equilibration steps underwent is 2000 moves, at an imaginary time-step set to 0.002 having a target weight of 1000 . The simulation took 10000 lines of data between accepted configurations. The calculated electric dipole moment from the output file is obtained at 11.29193731a.u. (which is the maximum distance from origin) with an acceptance ratio of $99.662 \%$ which is in good agreement as predicted by [31] and [19]. The results presented in Figure 1(a) and Figure 1(b) indicates that the more the DMC steps simulated, the more likely that the calculated the electric dipole moment value will be closer to the experimental value. The electric dipole moment value obtained from the DMC components is $2.0 \mathrm{D}$ with its symmetry directed along the $\mathrm{x}$-component as clearly shown in Figure 1.
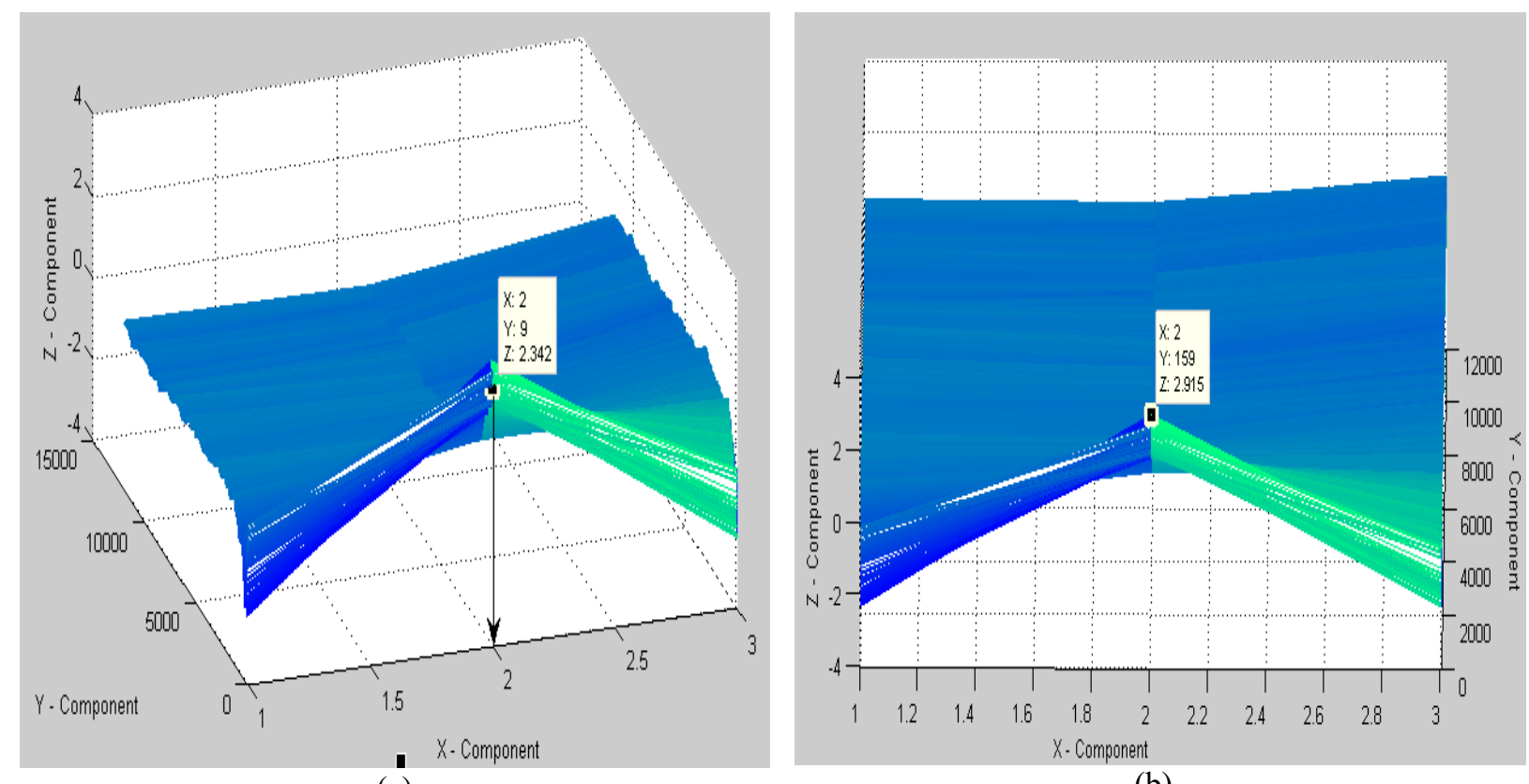

(a)

(b)

Figure 1: (a) Dorsal View, and (b) Lateral View, of DMC Components of the Electric Dipole Moment (in Debye) 
Thus, the electric dipole moment calculated in this work using CASINO-code compares favourably well with the experimental value of Seddon et al. [12]; and although it agrees perfectly-well with a previous VQMC study [32] as shown in Table 1, the DMC approach reached equilibration within few configuration steps unlike the VQMC. From our result, we observed that the deviation from experiment is $0.15 \mathrm{D}$ (equivalent to $8.1 \%$ ) which is highly reduced compared to the recent works of [13] and [2] which are $20.0 \%$ and $21.6 \%$ deviations away from the experimental value [12]. This result support claims that DMC provides near chemical accuracy as predicted by [19]. However, the deviation of the electric dipole moment in this study may be due to the single determinant SlaterJastrow trial wave-function used, since inadequacy in the Jastrow can increase the locality approximation bias. Nevertheless, Figure 2 shows a comparative analysis of the DMC technique with a previous VQMC study [32], showing the convergence in both methods and indicating instability in the VQMC method as its electric dipole moment square fluctuated above 120 within the numbers of steps. However, the standout points in the graph of Figure 2 may be due to inclusion of unequilibrated data in the final average data which will give a systematic bias to the averages obtained as predicted by [33]. The CASINO simulations from the DMC method shows a significant improvement in its stability towards the experimental value over the VQMC method as presented in the graph of Figure 2; and this could be attributed to the stochastic nature of the DMC method.

Table 1: Comparative analysis of the electric dipole moment (in Debye) of hydrazine molecule calculated by different researchers.

\begin{tabular}{llll} 
Authors & Year & Techniques / Methods & E.D.M. \\
\hline Seddon et al. [12] & 1976 & Experimental Value & 1.85 \\
Nelson Jr. et al.[11] & 1967 & Principal Methods & 1.75 \\
Kaczmarek et al.[13] & 2009 & Molecular Dynamics & 2.22 \\
Elts et al. [2] & 2014 & Molecular Model & 2.25 \\
Ekong et al. [32] & 2015 & VQMC & 2.00 \\
This work & & DMC (CASINO-code) & 2.00 \\
\hline
\end{tabular}

E.D.M. = Electric Dipole Moment

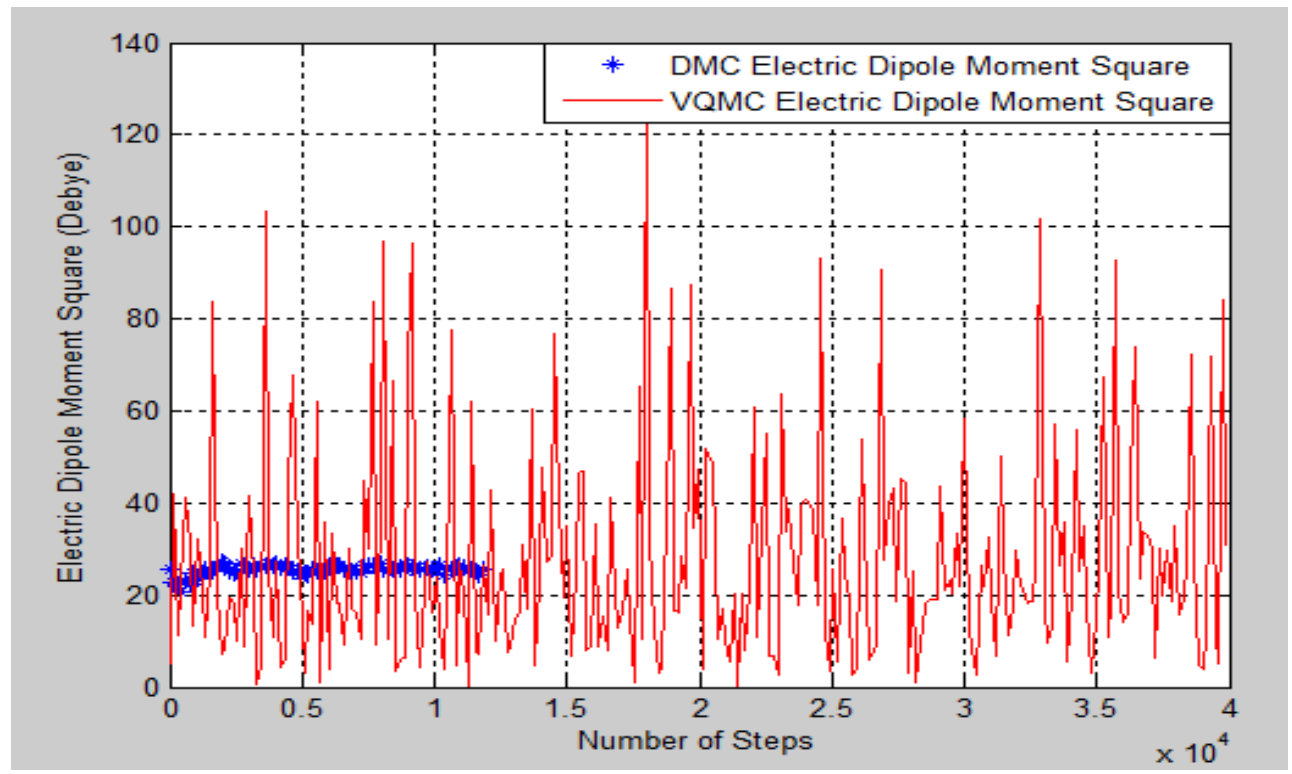

Figure 2: Graph of Electric Dipole Moment versus Number of VMC/DMC Steps

Similarly, the QMC CASINO-code was employed to calculate the ground-state total energy of hydrazine molecule using both the $\mathrm{VMC}$ and $\mathrm{DMC}$ techniques mentioned in section 2, and the results are presented in the graph of Figure 3 below. This shows that the more the number of steps 
simulated, the smaller the error bar. The ground-state total energies obtained from our simulations are $-111.68013 \pm 0.00891$ a.u. for VMC and $-111.842774 \pm 0.00394$ a.u. for DMC methods. This means that the electronic correlation effect is higher in DMC than the VMC. In this study, we found that our VMC result is in perfect agreement with the result obtained by [17] while the DMC result shows a variance in the ground-state total energy of hydrazine molecule as it gives a ground-state total energy lower than every other theoretical values in literature as shown in Table 2 below. This suggests that our calculated DMC ground-state total energy may be the exact-theoretical value for hydrazine molecule.

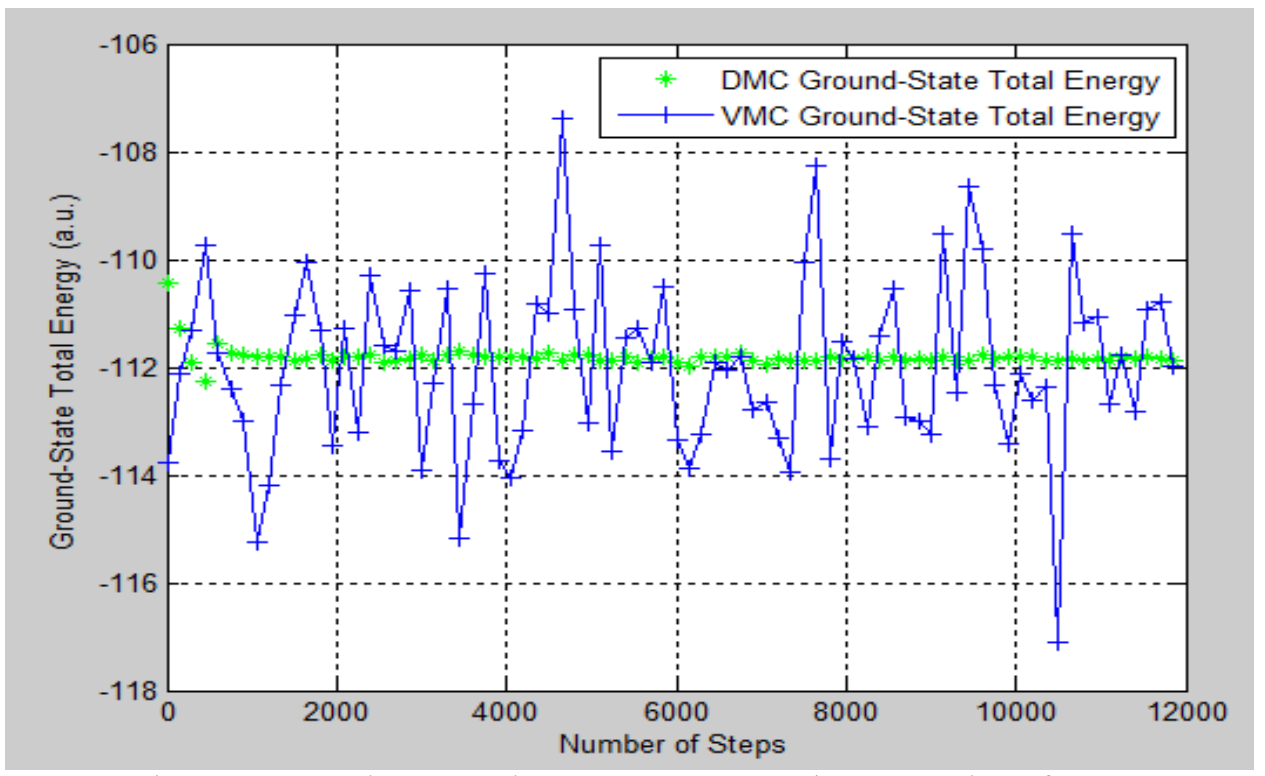

Figure 3: Ground-state total energy versus DMC/VMC number of steps

An observation from the output files is that the ground-state total energies were obtained at maximum distances of 9.02254018a.u. (for VMC) and 11.29193731a.u. (for DMC) from the origin, which falls within the limits of the theoretically obtained values. This indicates greater intensity of the lowest energy levels from configuration to equilibration at a small inter-particle distances. Also, from the output files, the result of the acceptance ratios of $51.979 \%$ (for $\mathrm{VMC}$ ) and $99.662 \%$ (for DMC) implies an improved stability in the ground-state total energy in the use of DMC over VMC methods. This indicates that the chosen time-step does not limit the number of accepted Monte Carlo move. Hence, as more configurations are included, the sampling is improved. The results of the ground-state total energy of hydrazine molecule calculated by different authors are summarized in Table 2.

Table 2: Comparative analysis of the ground-state total energy of hydrazine molecule (in a.u.) calculated by different researchers.

\begin{tabular}{llll} 
Authors & Year & Techniques / Methods & G.S.T.E. \\
\hline Alain Veillard [34] & 1966 & Non-empirical SCF-LCAO & -111.030 \\
Alagona [16] & 1991 & SCC-MO & -111.238 \\
Alagona [16] & 1991 & Ab initio DZ & -111.226 \\
Peel [15] & 1986 & HF/MP2/6-31G* & -111.5544 \\
Schlegel and Skancke[17] & 1993 & Perturbation & -111.68045 \\
Pires and Jorge [18] & 2005 & IGCHF & -111.22978 \\
This work & & VMC (CASINO-Code) & -111.68013 \\
This work & & DMC (CASINO-Code) & -111.84277 \\
\hline
\end{tabular}

G.S.T.E. $=$ Ground-state Total energy

1 a.u. $=27.2 \mathrm{eV}$ 


\section{CONCLUSION}

With the QMC-CASINO package, the electric dipole moment and the ground-state total energy of hydrazine molecule were calculated from both DMC and VMC methods in CASINOcode. The results obtained show that the electric dipole moment of the hydrazine molecule calculated with DMC differs from the experimental value with only $8.1 \%$ compared to other values in literature. Also, while the ground-state total energy of hydrazine molecule obtained from the VMC method is -111.68013 a.u., the DMC gave a value of -111.84277 a.u. which is -0.16232 a.u. (i.e. $-0.145 \%$ ) below the lowest value calculated by [17], suggesting that this could be the exact ground-state total energy of $\mathrm{N}_{2} \mathrm{H}_{4}$ molecule. The results obtained show that DMC technique can give a more accurate ground-state result with very high accuracy.

\section{ACKNOWLEDGMENTS}

We have benefited from the exchange of ideas, materials, and results with other colleagues, to whom we offer our thanks. We thank Richard Needs, Mike Towler, Neil Drummond, and Pablo López Ríos, of the Theory of Condensed Matter Group, Cavendish Laboratory, J. J. Thomson Avenue, Cambridge, United Kingdom, for the release of the CASINO-code.

\section{References}

[1] Skurski, P., M. Gutowski, and J. Simons, (1999): J. Phys. Chem. A, 103, Pp. 625.

[2] Elts, E., T. Windmann, D. Staak and J. Vrabec, Fluid phase behavior from molecular simulation: Hydrazine, Monomethylhydrazine, Dimethylhydrazine and Binary mixtures containing these compounds, Pp. 3 and 6. [Unpublished]

[3] Collins, G. E., and S. L. Rose-Pehrsson, (1994), Fluorescent detection of hydrazine, monomethylhydrazine, and 1,1-dimethylhydrazine by derivatization with aromatic dicarbaldehydes, Analyst 119, Pp. 1907-1913.

[4] Kallikragas, D. T., K. I. Choudhry, A. Y. Plugatyr, and I. M. Svishchev, (2013), Diffusivity and hydration of hydrazine in liquid and supercritical water through molecular dynamics simulations and split-flow pulse injection experiments, The Journal of Chemical Physics 139, Pp. 134507.

[5] Syage, J. A., R. B. Cohen, and J. Steadman, (1992), J. Chem. Phys. 97: 6072.

[6] Sahebalzamani, H., F. Salimi, and E. Dornapour (2013), Theoretical Studies of Structure, Spectroscopy, and Properties of a New Hydrazine Derivative, Journal of Chemistry, Volume 2013, Article ID 187974, Pp.1

[7] Gutowski, K. E., B. Gurkan, and E. J. Maginn, (2009), Pure Appl. Chem. 81, Pp. 1799-1828.

[8] Ueda, S., Y. Kuroda, H. Miyajima, and T. Kuwara, (1994): J. Prop. Power 10: Pp. 646.

[9] Agusta, M. K., M. David, H. Nakanishi, and H. Kasai, (2010), Surface Science 604: Pp. 245.

[10] Von Burg, R., and T. Stout, (1991), J. Appl. Toxicol; 11, Pp. 447 - 450.

[11] Nelson Jr., R. D., D. R. Lide Jr., and A. A. Maryott, (1967), Selected values of electric dipole moments for molecules in the gas phase, National Standard Reference Data Series [NSRDS]. National Bureau of Standards 10

[12] Seddon, W. A., J. W. Fletcher and F.C. Sopchyshyn, (1976), Can. J. Chem. 54, Pp. 28072812.

[13] Kaczmarek, A., M. Shiga, and D. Marx, (2009): J. Phys. Chem. A 113, Pp. 1985-1994.

[14] Yamabe, H., H. Kato and T. Yonezawa, (1971), Bulletin of the Chemical Society of Japan, Vol. 44, Pp. 22-27. 
[15] Peel, J. Barrie, (1986), J. Chem., 39, Pp. 1284.

[16] Alagona, G, (1991), Theoretical Model of Chemical Bonding, Part 3, Molecular Spectroscopy, Electronic Structure and Intramolecular Interactions, Edited by Z. B. Maksic, Pp. 278.

[17] Schlegel, H. B. and A. Skancke, (1993): J. Am. Chem. Soc., Vol. 115, No. 16, Pp. 7466.

[18] Pires, J. M. and F. E. Jorge, (2005), Indian Journal of Chemistry, Vol. 44A, Pp. 1979.

[19] Grossman, J. C., (2002), Benchmark quantum Monte Carlo calculations, Journal of Chemical Physics Volume 117, Number 4, Pp. 1434.

[20] Foulkes, W. M. C., L. Mitas, R. J. Needs, and G. Rajagopal, (2001), Rev. Mod. Phys., 73, Pp. 33.

[21] Reynolds, P. J., D. M. Ceperley, B. J. Alder, and W. A. Lester Jr., (1982), Fixed-node quantum Monte Carlo for molecules, J. Chem. Phys. 77, Pp. 5593-5603.

[22] Anderson, J. B., (1995), Fixed-node quantum Monte Carlo, Int. Rev. Phys. Chem. 14, Pp. 85112.

[23] Needs, R. J., M. D. Towler, N. D. Drummond, and P. López Ríos, (2009), CASINO user's guide, version 3.0.0 (University of Cambridge, UK, 2009)

[24] Roothaan, C. C. J., (1960), Self Consistent Field Theory for Open Shell of Electronic Systems, Rev. Mod. Phys. 32 (2): 179-185.

[25] Lee, M. A., P. Vashista, and R. K. Kalia, (1983): Phys. Rev. Lett. 129, Pp. 2422.

[26] Mohan, V., and J. B. Anderson, (1989): Chem. Phys. Lett. 156, Pp. 520.

[27] Lüchow, A. and J. B. Anderson, (2000): Monte Carlo Methods in Electronic Structures for Large Systems. Annual Review of Physical Chemistry, Vol. 51: 501-526.

[28] Metropolis, N., A. W. Rosenbluth, M. N. Rosenbluth, A. M. Teller, and E. Teller, (1953), J. Chem. Phys. 21: 1087-1092.

[29] Kalos, M. H., and P. A. Whitlock, (1986), In Monte Carlo Methods, New York: Wiley, Pp. 73-86.

[30] Umrigar, C.J., J.G. Wilson, K.W. Wilkins, (1988), Optimized trial wave functions for quantum Monte Carlo calculations, Phys. Rev. Lett. 60, Pp. 1719-1722.

[31] Needs, R. J., M. D. Towler, N. D. Drummond, and L. P. Rios, (2012), Theory of Condensed Matter Group, Cavendish Laboratory, Cambridge, UK. CASINO User's Guide, Version 2.11.0.

[32] Ekong, S. A., V. A. Akpan and D. A. Oyegoke, (2015), Nig. J. of Pure and Appl. Physics, Vol. 6, No. 1, Pp. $1-5$.

[33] Kent, P. R. C., (1999): Techniques and Applications of Quantum Monte Carlo, Ph.D - Thesis at the University of Cambridge.

[34] Alain Veillard, (1966), Theoretica chimica acta, Volume 5, Issue 5, Pp. 413. 